\title{
A new statistical method for design and analyses of component tolerance
}

\author{
Mohammad Mehdi Movahedi $^{1} \cdot$ Mohsen Khounsiavash $^{2} \cdot$ Mahmood Otadi $^{3}$ • \\ Maryam Mosleh ${ }^{3}$
}

Received: 14 November 2015/Accepted: 23 August 2016/Published online: 6 September 2016

(C) The Author(s) 2016. This article is published with open access at Springerlink.com

\begin{abstract}
Tolerancing conducted by design engineers to meet customers' needs is a prerequisite for producing highquality products. Engineers use handbooks to conduct tolerancing. While use of statistical methods for tolerancing is not something new, engineers often use known distributions, including the normal distribution. Yet, if the statistical distribution of the given variable is unknown, a new statistical method will be employed to design tolerance. In this paper, we use generalized lambda distribution for design and analyses component tolerance. We use percentile method (PM) to estimate the distribution parameters. The findings indicated that, when the distribution of the component data is unknown, the proposed method can be used to expedite the design of component tolerance. Moreover, in the case of assembled sets, more extensive tolerance for each component with the same target performance can be utilized.
\end{abstract}

Keywords Nonlinear programming - Generalized lambda distribution (GLD) · Tolerancing · Percentile matching estimates

This article is extracted from a research plan that is done by research budget of Islamic Azad University, Firoozkooh Branch, Firoozkooh, Iran.

Mohammad Mehdi Movahedi

m_m_movahedi@yahoo.com

1 Department of Management, Firoozkooh Branch, Islamic Azad University, Firoozkooh, Iran

2 Department of Electrical, Biomedical and Mechatronics Engineering, Qazvin Branch, Islamic Azad University, Qazvin, Iran

3 Department of Mathematics, Firoozkooh Branch, Islamic Azad University, Firoozkooh, Iran

\section{Introduction}

In mass production, products are assembled using components processed or manufactured by different machines or processes. This requires all the components to be replaced at the time of assembling. In addition, given the changes applied in machines, tools, human resources, raw materials, and production methods, the quality characteristics (e.g. length, diameter, tensile strength) will always be subject to change as well. Given the inevitability of changes as well as the necessity of being replaceable, the permissible limits, called tolerance, need to be determined according to the changes in quality characteristics. Customers and product designers are the factors that determine the tolerance of quality characteristics of the assembled product based on the operational requirements provided by the customers. Then, this tolerance should be allocated to the quality characteristics of the assembled components.

Tolerance can be defined as the physical or chemical properties (e.g. size, weight, strength and the combination of components) or geometric characteristics (e.g. dimensions, position, shape and surface finish of some part features). As one cannot manufacture many components with the same nominal value, the deviation from nominal value will be inevitable. That is why tolerance is allowed. If a component has a high deviation from the nominal value, its quality will suffer. Consequently, the design engineers define the maximal permissible specification limits, called tolerance, with the purpose of hampering the degradation in the performance of the product (Devor et al. 2007).

Specification limits, or conformance boundary specified for a characteristic, have been defined in Standard ISO as those limits of technical or design characteristics within which limits are specified for products or services. These limits usually match customer requirements and can be 
either two way with upper and lower limits or one way with either upper or lower limit. Sometimes, the limits of technical characteristics are determined based on the distribution of quality characteristics. For example, some statistics derived from individual units can be used. Standard tolerance limits will accelerate the process of design (Wadsworth et al. 2002). Therefore, this study will consider the limits of technical characteristics, as defined above.

Standard tolerance limits can be obtained through conducting statistical calculations based on one or more samples of an assumed quality characteristic. As a result, this can differ from the specification limits, which are the requirements for individual components. If the design consideration is not enough to determine the technical specifications of each component, some criteria for process capability will be potentially used on that purpose. Tolerance determination plays a significant role in improvement of Six Sigma as well as the validation of the process capability calculation.

Tolerance is the difference between the upper and lower limits of the technical characteristics of a product. Let the tolerance of $X_{i}$ be $T_{i}, i=1,2, \ldots, k$, and let the tolerance of the assembly characteristic $X$ be $T$; then in general, for any linear function of $X$, if

$X=X_{1} \pm X_{2} \pm X_{3} \pm \cdots \pm X_{k}$

it can be

$T=T_{1}+T_{2}+\cdots+T_{k}$.

This equation is called additive relationship, which can be used by a design engineer to allocate the tolerances $T_{1}, \ldots, T_{k}$ for an assumed technical characteristic $T$.

Considering that relations depend on the characteristic of components as well as the assembled set of characteristics, the following relational assumptions should be put forward for tolerance determination (Chandra 2001).

(1) $X_{i}$ s are mutually independent.

(2) The components are selected and assembled randomly.

(3) $X_{i} \sim N\left(\mu_{i}, \sigma_{i}^{2}\right)$.

(4) The process of $X_{i}$ is under control, and the mean of $X_{i}$ distribution, that is, $\mu_{i}$, is equal to the best value of $X_{i}$ and is the midpoint of the valid range of $X_{i}$. Then,

$\mu_{i}=\frac{\left(U_{i}-L_{i}\right)}{2}$.

(5) The standard deviation of $X_{i}$ characteristic is produced by the process in such a way that $99.73 \%$ of the $X_{i}$ characteristic will fall within the range of its characteristics.

Then based on the above-mentioned assumptions, the tolerance of the component $X_{i}$ can be calculated as follows;
$U_{i}-L_{i}=T_{i}=6 \sigma_{i}, i=1,2, \ldots, k$

Supposing $\mu$ and $\sigma^{2}$ are the mean and variance of $X$, respectively, and $X=X_{1} \pm \cdots \pm X_{k}$, then

$\mu=\mu_{1} \pm \mu_{2} \pm \mu_{3} \pm \cdots \pm \mu_{k}$

as Xis are mutually independent; then,

$\sigma^{2}=\sigma_{1}^{2}+\sigma_{2}^{2}+\sigma_{3}^{2}+\cdots+\sigma_{k}^{2}$.

Taking into consideration the second assumption, the assembled characteristic set $X$ has a normal distribution. If $99.73 \%$ of all assembled characteristic set $X$ falls between the characteristic range $U$ and $L$, Eq. (4) will be derived as follows:

$(U-L)=T=6 \sigma$.

Given the Eqs. (4) and (7), we will have

$\sigma_{i}^{2}=\left(\frac{T_{i}}{6}\right)^{2}, i=1,2, \ldots, k$

and

$\sigma^{2}=\left(\frac{T}{6}\right)^{2}$

The combination of Eqs. (6), (8), and (9) will yield

$\left(\frac{T}{6}\right)^{2}=\left(\frac{T_{1}}{6}\right)^{2}+\left(\frac{T_{2}}{6}\right)^{2}+\cdots+\left(\frac{T_{k}}{6}\right)^{2}$

or

$T=\sqrt{T_{1}^{2}+T_{2}^{2}+\cdots+T_{k}^{2}}$.

Equation (11), called a probable relation, provides another concept for allocating tolerance to the components of an assembled set for the assumed tolerance. It can be seen that the tolerance of each component can arise, if we use the probability relation (Chandra 2001).

In this study, we will use generalized lambda distribution (GLD) to determine the tolerance of the components whose probability distribution function is unknown.

Literature review and background are presented below, followed by the introduction of generalized lambda distribution and its parameter estimation method. Then, the use of this method will be illustrated by an example.

\section{Literature review and background}

The manufacturing and production costs reduce as the tolerance of quality characteristics increase. On the other hand, as mentioned in the introduction, all things being equal, a higher tolerance can be defined using a probability method. 
Ginsberg (2013) presents an optical design method and flowchart that emphasize considering component tolerances early in the design process. The paper discusses the proposed process by using it for designing two different optical systems. The author demonstrates the process of developing sensitivity tables and how to apply them to develop a tolerance budget.

Zhang and Hook (1992) argue that the main problem is the control of sequential tolerance, which is appropriate for those components moving in a series of consequential processes and using real-time criteria for consecutive manufacturing. They choose the target point of machine operation to maximize the output quality.

Wheeler et al. (1999) have developed a probability method to select an optimal sub-set of technology-based processes required for implementing a process design under the control strategy of traditional tolerance. They present an implicit enumeration approach to the selection of an optimum subset of technological processes required to execute a process plan under a conventional tolerance control strategy. They present a probabilistic approach to the problem and use the first-order second moment method (FOSMM) to estimate the yield for an interdependent system of functional requirements.

Chandra (2001) uses uniform, normal, and beta distributions to determine the tolerance. He also utilizes linear planning as well as nonlinear relations. Finally, he has proposed the use of dynamic planning for such a purpose, and reviewing the tolerance determination techniques.

Devor et al. (2007) use loss function to determine the tolerance. Through quantifying the loss function, they assert that tolerance should be determined to minimize the loss function and contend that the first attempt to reduce the quality costs (including the costs associated with defective components, re-working and other costs) focuses on decreasing the costs related to defective components. They have also applied different statistical distributions to determine the component tolerance.

Sampath Kumar et al. (2009) strived to investigate the optimal tolerance allocation by considering both tolerances and manufacturing cost, so that the total assembly cost is minimized. A new global nonlinear optimization technique called Pattern search algorithm has been executed to figure the optimal tolerance allocation and asymmetric total cost to overcome the defects in the traditional tolerance allocation problem.

Macko et al. (2012) describe a negative effect of size tolerance on the trigger characteristic. The trigger characteristic is defined as the dependence of trigger force and trigger angle. Their study indicates an example of trigger mechanism that is designed as a Glock type of mechanism. The authors proposed the use of software MW as the solution for designers of small arms.
George (2012) further contributed to the research by concentrating on the kind of tolerances that are broadly applied in industry and reverse engineering. To the best of their knowledge, the authors argue that their approach is an initial attempt to solve this type of RE problems that can be directly implemented within a CAD environment.

Hasenauer (2013) and Shannon (2013) have used an optical technique instead of a mechanical one to determine the tolerance. The optical technique that is a more complex one generally takes into account dimensional changes of the components. However, the permissible changes are determined, using the effects of a complete set of waves passing through the lens.

Nili Ahmadabadi et al. (2012) proposed a five-parameter generalized lambda distribution for process control. To estimate the parameters of distribution, they use the moment-matching method proposed by Ramberg and Schmeiser (1974).

Armillotta (2015) proposes a method for tolerance analysis on planar structures and mechanisms. The fundamental deficit of the actual tolerancing and specification systems is illustrated by Weckenmann and Hartmann (2015). For tolerancing of a component, Yu and Lub (2016) use quality-oriented statistical tolerancing (ST) technique, which helped to overcome the challenges of modern manufacturing. Jean-Marc (2016) show that statistical tolerancing becomes risky when idealized centering assumptions are not perfectly achieved. Alain Van Hoecke (2016) introduced risk tool in statistical tolerancing and its verification management to optimize customers' and suppliers' risks. He shows that tolerancing-verification coupling increases benefits by enlarging tolerances through risk control.

\section{Generalized lambda distribution (GLD)}

Generalized lambda distribution was initially proposed by Tukey (1962) and developed by Joiner and Rosenblatt (1971). This distribution can fit the common distributions such as normal, lognormal, Weibull, etc. with high accuracy. It is also able to fit in with continuous distributions; the flexibility of this type of distribution is compatible with data histograms and the estimation of the kind of their distribution. As a result, GLD can be used as a powerful instrument for conducting research in various areas including the estimation of parameter, adapting distributions with the data as well as the simulation research which is based on data production. For example, it can be applied in meteorology (Ozturk and Dale 1982), queue systems (Dengiz 1988), psychology (Delaney and Vargha 2000), operation research (Ganeshan 2001), corrosion (Najjar et al. 2003), equipment defect and fatigue (Bigerelle et al. 
2006), statistical quality control (Fournier et al. 2006), engineering (Rochan et al. 2008), and reliability (Movahedi et al. 2013).

Tukey's (1962) lambda family of distributions is defined by the quantile function $Q(p)$ :

$Q(p)=\left\{\begin{array}{ll}\frac{p^{\lambda}-(1-p)^{\lambda}}{\lambda}, & \lambda \neq 0 \\ \frac{\log (p)}{1-p} & , \lambda=0\end{array}\right.$,

where $p$ denotes the probabilities, $p \in[0,1]$. Ramberg and Schmeiser (1974) developed four-parameter quantile function lambda distribution as below:

$$
\begin{aligned}
F^{-1}(\mathrm{p} ; \lambda) & =F^{-1}\left(p ; \lambda_{1}, \lambda_{2}, \lambda_{3}, \lambda_{4}\right) \\
& =\lambda_{1}+\frac{p^{\lambda_{3}}-(1-p)^{\lambda_{4}}}{\lambda_{2}},
\end{aligned}
$$

where $p$ denotes the probabilities, $p \in[0,1], \lambda_{1}$ and $\lambda_{2}$ indicate the location and scale parameters, and $\lambda_{3}$ and $\lambda_{4}$ denote the shape parameters jointly associated with the strengths of the lower and upper tails, respectively. In the limiting case $\lambda_{1}=0$ and $\lambda_{2}=\lambda_{3}=\lambda_{4}=\lambda$, Tukey's (1962) lambda distribution will be obtained.

Ramberg et al. (1979) note that the proposed distribution (Eq. 13) is not defined for certain combinations of the parameters.

In this section, we will describe the properties of a fiveparameter generalization of the lambda distribution to obtain smoothed analytic representations for grouped data (Tarsitano 2005). The GLD is determined by the quantile function

$X(p, \lambda)=\lambda_{1}+\lambda_{2} p^{\lambda_{4}}-\lambda_{3}(1-p)^{\lambda_{5}}, \quad 0 \leq p \leq 1$,

where $\lambda_{1}$ denotes a location parameter $\lambda_{2}$ and $\lambda_{3}$ shows linear parameters prevalently concerning the scale of the variable, and $\lambda_{4}$ and $\lambda_{5}$ are exponential parameters determining the shape of the quantile function.

Expression (14) readily produces $X(p, \lambda)$ in terms of a uniform random variable $p$ on the interval $[0,1]$. This fact is particularly relevant not only for the simulation experiment, but also for order statistics, optimal grouping, inequality measures, heavy tail behavior analysis, loss distributions, oscillatory interpolation, and Q-Q plotting.

Analytic expression for the cumulative distribution function $F(x, \lambda)$ of a GLD model is in general not available. However, the fact that the GLD is not invertible is not a serious drawback, because the same is true for many popular models such as normal, lognormal, generalized gamma, and generalized beta. The GLD is observed to fit, by suitable choice of $\lambda$, to various theoretical and practical distributions (Karian and Dudewicz 1999, 2000).
There are several parameterizations of the asymmetric lambda distribution and all the versions can be obtained as special cases of (14) (Shapiro and Wilk (1965), Joiner and Rosenblatt (1971), Ramberg and Schmeiser (1974), Filliben (1975), Hoaglin (1975), Lam et al. (1980), Freimer et al. (1988), Sarabia (1996), Devroye (1996), Gilchrist (2000)).

\section{Parameter estimation}

In the review of literature, various methods for estimating generalized lambda distribution parameters have been introduced, for instance, moment-matching method, least squares method, Starship method and Downhill simplex method. Similarly, the percentile matching (PM) estimates, moment matching (MM) estimates, probability-weighted moment (PWM) estimates, minimum Cramér-Von Mises (MCM) estimates, maximum likelihood (ML) estimates and pseudo least squares (PLS) estimates have been employed by Tarsitano (2005) to estimate the parameters of five-parameter lambda distribution. In addition, Nasser and Aljazar (2005) estimate the parameters of four-parameter lambda distribution using the moments of the GLD and the least square method. Fournier et al. (2007) used the percentile method to estimate the parameters of four-parameter lambda distribution.

Tarsitano (2005) pointed out that the percentile matching (PM) estimation method is advantageous over other methods. First and foremost, less weight is assigned to the outliers in this method than in the moment estimates, and the PM estimators can still be calculated when moments do not exist. Second, the validity of this method is seriously restricted because of the lack of a theoretical justification in the selection of a specific set of percentiles. Third, the sextiles utilized in our experiments have brought about adequate results for symmetric distributions.

In this article, we have used PM estimates introduced by Tarsitano (2005) adapted from the methods developed by Schmeiser and Deutsch (1977), Harrell and Davis (1982), and Korn et al. (1997). In the rest of the paper, this method will be briefly introduced (see Tarsitano, 2005).

For this method, it is presumed that all $n$ observations are from the same parametric distribution. Supposing that a sample of size $n$ from a GLD is grouped into k intervals, then

$$
\begin{aligned}
\left(X_{i-1}, X_{i}\right], \quad n_{i}, \quad N_{i} & =\sum_{j=1}^{i} n_{j} ; f_{j}=\frac{n_{j}}{n}, \quad p_{i}=\sum_{j=1}^{i} f_{j}, \quad i \\
& =1,2, \ldots, k .
\end{aligned}
$$


The values $\left\{X_{i}, i=0,1,2, \ldots, k ; X_{i-1}<X_{i}\right\}$ indicate the limits of $k$ exhaustive and non-overlapping classes and $k \geq 3$ is limited in effect. We can write the probability mass attributed to the ith class as

$$
\begin{aligned}
F\left(X_{i} ; \lambda\right)-F\left(X_{i-1} ; \lambda\right) & =\pi_{i}(\lambda)>0, i \\
& =1,2, \ldots, k ; \sum_{i=1}^{k} \pi_{i}(\lambda)=1 .
\end{aligned}
$$

It is assumed that $\lambda \in \Lambda \subset R^{5}$ and we consider $\lambda \in \Lambda$ as the true, but unknown value of $\lambda$. percentile matching (PM) estimates method, which consists of equating a selection of five empirical $X_{i}$ and five theoretical percentiles $X\left(p_{i}, \lambda\right)$. If $X_{i}$ is a good approximation of the unknown empirical order statistic corresponding to $p_{i}$, then

$X_{i_{j}}=\lambda_{1}+\lambda_{2} p_{i_{j}}^{\lambda_{4}}-\lambda_{3}\left(1-p_{i_{j}}\right)^{\lambda_{5}} ; j=1, \ldots, 5$,

where $i_{j} \in\left(1,2, \ldots, k^{\prime}\right)$ and $k^{\prime}=k$ if the upper limit of the variable is known and finite otherwise $k^{\prime}=k-1$.

To sustain the computation at a reasonable level, the PM estimates were obtained by applying system (14) to the five sextiles $w_{1}, w_{2}, w_{3}, w_{4}, w_{5}$ computed by

$$
\begin{aligned}
w_{s} & =\left(1-\beta_{s}\right) X_{j-1}+\beta_{s} X_{j} ; \beta_{s}=\frac{p_{s}-p_{j-1}}{f_{j}}, p_{s}=\frac{s}{6}, j \\
& =\underset{1 \leq i \leq k}{\operatorname{Min}}\left\{p_{i} \geq p_{s}\right\}, s=1,2, \ldots, 5 .
\end{aligned}
$$

Because (18) is a nonlinear system of equations in $\lambda$, an iterative procedure needs to be employed for solving $\lambda$. We have applied the downhill simplex minimization to the following criterion to calculate the PM estimates:

$S_{\mathrm{QM}}(\lambda)=\operatorname{Max}_{1 \leq s \leq 5}\left\{\left|w_{s}-X\left(p_{s}, \lambda\right)\right|\right\}$

under the constraint that $\min \left(\lambda_{4}, \lambda_{5}\right)>-1$, because Tarsitano (2005) indicates that the $i$ th moment of the GLD holds true if and only if $\min \left(\lambda_{4}, \lambda_{5}\right)>-1$.

Finally, having determined the five parameters of the distribution, we can demonstrate that the mean and variance of GLD can be calculated as follows:

$$
\begin{aligned}
\mu & =\lambda_{1}+\frac{\lambda_{2}}{\left(\lambda_{4}+1\right)}-\frac{\lambda_{3}}{\left(\lambda_{5}+1\right)}, \\
\sigma^{2} & =\int_{0}^{1}\left(\lambda_{1}+\lambda_{2} p^{\lambda_{4}}-\lambda_{3}(1-p)^{\lambda_{5}}\right) \mathrm{d} p-\left(\lambda_{1}+\frac{\lambda_{2}}{\left(\lambda_{4}+1\right)}-\frac{\lambda_{3}}{\left(\lambda_{5}+1\right)}\right)^{2},
\end{aligned}
$$

$\sigma=\sqrt{\int_{0}^{1}\left(\lambda_{1}+\lambda_{2} p^{\lambda_{4}}-\lambda_{3}(1-p)^{\lambda_{5}}\right) \mathrm{d} p-\left(\lambda_{1}+\frac{\lambda_{2}}{\left(\lambda_{4}+1\right)}-\frac{\lambda_{3}}{\left(\lambda_{5}+1\right)}\right)^{2}}$
Using this method, we can take enough random samples from the production line and then measure the generalized lambda parameters. Finally, the tolerance of the components is designed through the calculation of distribution variance and mean.

\section{Data collection and results}

In this section, we assume an assembled set containing two target values: 14 and 16, respectively. Then, two random 100-digit series representing the thickness of each component is produced, using MATLAB software. The frequency distribution table is drawn up, using Eq. 15. Then, the distribution parameters are estimated, using Eqs. 1619.

$\chi^{2}$ test can be used to make sure that the answers are correct. Further, the mean, variance, and standard deviation are measured using Eqs. 20 and 21. Finally, the tolerances of the component are determined.

Tables 1 and 2 display the results of the characteristics of 1000 random samples for two components with target values 14 and 16 based on Eq. 15 .

Now, the relations can be based on Eqs. (17), (18), and (19). Equation 19 can be solved for the first and second

Table 1 Random data for the first component with target value $14 \mathrm{~mm}$

\begin{tabular}{lrrll}
\hline Ranges & \multicolumn{1}{l}{$n_{i}$} & $N_{i}$ & $f_{i}$ & $p_{i}$ \\
\hline $13.95-13.97$ & 185 & 185 & 0.185 & 0.185 \\
$13.97-13.99$ & 235 & 420 & 0.235 & 0.420 \\
$13.99-14.01$ & 195 & 615 & 0.195 & 0.615 \\
$14.01-14.03$ & 180 & 795 & 0.180 & 0.795 \\
$14.03-14.05$ & 190 & 985 & 0.190 & 0.985 \\
14.05 and more & 15 & 1000 & 0.015 & 1.000 \\
Total & 1000 & - & 1.000 & - \\
\hline
\end{tabular}

Table 2 Random data for the second component with target value $16 \mathrm{~mm}$

\begin{tabular}{lrrll}
\hline Ranges & \multicolumn{1}{l}{$n_{i}$} & $N_{i}$ & $f_{i}$ & $p_{i}$ \\
\hline $15.95-15.97$ & 170 & 170 & 0.170 & 0.170 \\
$15.97-15.99$ & 205 & 375 & 0.205 & 0.375 \\
$15.99-16.01$ & 265 & 640 & 0.265 & 0.640 \\
$16.01-16.03$ & 180 & 820 & 0.180 & 0.820 \\
$16.03-16.05$ & 170 & 990 & 0.170 & 0.990 \\
16.05 and more & 10 & 1000 & 0.010 & 1.000 \\
Total & 1000 & - & 1.000 & - \\
\hline
\end{tabular}


Table 3 Chi-square test for the first component

\begin{tabular}{llllll}
\hline $\begin{array}{l}\text { Class } \\
\text { interval }\end{array}$ & $\begin{array}{l}\text { Observation } \\
\text { value }\left(O_{i}\right)\end{array}$ & $\begin{array}{l}\text { Cumulative relative } \\
\text { frequency }\left(Y_{i}\right)\end{array}$ & $\begin{array}{l}\text { Expected observation } \\
\text { value }\left(E_{i}\right)\end{array}$ & $\left(O_{i}-E_{i}\right)^{2}$ & $\frac{\left(O_{i}-E_{i}\right)^{2}}{E_{i}}$ \\
\hline 1 & 13.96 & 0.185 & 12.86 & 1.210 & 0.0941 \\
2 & 13.98 & 0.420 & 13.40 & 0.336 & 0.0251 \\
3 & 14.00 & 0.615 & 14.20 & 0.040 & 0.0028 \\
4 & 14.02 & 0.795 & 15.22 & 1.440 & 0.0946 \\
5 & 14.04 & 0.985 & 16.62 & 6.656 & 0.4005 \\
6 & 14.06 & 1.000 & 16.75 & 7.236 & 0.4320 \\
Total & & & & & \\
\hline
\end{tabular}

Table 4 Chi-square test for the second component

\begin{tabular}{llllll}
\hline $\begin{array}{l}\text { Class } \\
\text { interval }\end{array}$ & $\begin{array}{l}\text { Observation } \\
\text { value }\left(O_{i}\right)\end{array}$ & $\begin{array}{l}\text { Cumulative relative } \\
\text { frequency }\left(Y_{i}\right)\end{array}$ & $\begin{array}{l}\text { Expected observation } \\
\text { value }\left(E_{i}\right)\end{array}$ & $\left(O_{i}-E_{i}\right)^{2}$ & $\frac{\left(O_{i}-E_{i}\right)^{2}}{E_{i}}$ \\
\hline 1 & 15.96 & 0.170 & 16.49 & 0.54 & 0.2916 \\
2 & 15.98 & 0.375 & 16.00 & 0.02 & 0.0004 \\
3 & 16.00 & 0.640 & 15.70 & 0.30 & 0.0900 \\
4 & 16.02 & 0.820 & 15.78 & 0.26 & 0.0676 \\
5 & 16.04 & 0.990 & 16.02 & 0.02 & 0.0004 \\
6 & 16.06 & 1.000 & 16.03 & 0.03 & 0.0009 \\
Total & & & & & 0.4509 \\
\hline
\end{tabular}

components, using MATLAB. Solving the problem through MATLAB leads to the following solutions.

First component: the values of the GLD parameters for the first component are as follows:

$$
\begin{aligned}
\lambda_{1} & =12.7436, \lambda_{2}=4.0029, \lambda_{3}=2.1856, \lambda_{4} \\
& =2.0851, \lambda_{5}=52.1962 .
\end{aligned}
$$

As a result, the quantile function of the first component is as follows:

$$
\begin{aligned}
X(p, \lambda)= & 12.7436+4.0029 p^{2.0851}-2.1856(1 \\
& -p)^{52.1962}, \quad 0 \leq p \leq 1 .
\end{aligned}
$$

Second component: the values of the GLD parameters for the second component are as follows:

$$
\begin{aligned}
\lambda_{1} & =9.2427, \lambda_{2}=6.7886, \lambda_{3}=-4.3945, \lambda_{4} \\
& =0.2331, \lambda_{5}=2.5099 .
\end{aligned}
$$

As a result, the quantile function of the first component is as follows:

$$
\begin{aligned}
X(\mathrm{p}, \lambda)= & 9.2427+6.7886 p^{0.2331}+4.3945(1 \\
& -p)^{2.5099}, \quad 0 \leq p \leq 1 .
\end{aligned}
$$

Goodness of fitness test: the second rank equations has more than one acceptable solution. Thus, it is necessary to run the goodness-of-fit test to make sure that the answers are correct. To this end, the Chi-square technique is used. $\chi_{o}^{2}=\sum_{i=1}^{k} \frac{\left(O_{i}-E_{i}\right)^{2}}{E_{i}}$.
$H_{0}$ and $H_{1}$ are set forward as follows:

$H_{0}$ : GLD where the obtained parameters fit the data.

$H_{1}$ : GLD where the obtained parameters do not fit the data.

If $\chi_{o}^{2}>\chi_{\alpha, k-i-1}^{2}$ where $\alpha$ is the level of significance of the test, $k$ the number of sets, and $\mathrm{i}$ the number of distribution parameters, $\mathrm{H}_{0}$ is rejected.

To conduct goodness-of-fit test for each part of the component, we should first obtain the expected values $\left(E_{i}\right)$. As for each set $i$ th, the cumulative amount of relative frequency is placed in the distribution relationship; thus, we calculate the value of the mean of the given set, i.e., $E_{i}$. In the same way, the mean of the data set is in fact the observed mean. The Chi-square technique can test with $K-6$ degrees of freedom, where $K$ is the number of class intervals. Tables 3 and 4 shows the hypothesis testing of the research data.

The relationship for the first component can be expressed as $\chi_{o}^{2}(1.0491)<\chi_{o}^{2}(3.84)$, and for the second component as $\chi_{o}^{2}(0.4509)<\chi_{o}^{2}(3.84)$. Hence, we can conclude that for both components the derived generalized lambda distribution fits the data.

\section{Design of the tolerance}

In this section, to design tolerance we must first determine the mean and standard deviation for both components. 
Thus, we receive them from Eqs. 20, 21, and 22. For the first component they will be:

$\mu_{1}=14, \sigma_{1}^{2}=0.00049$ and $\sigma_{1}=0.022$,

and for the second component:

$\mu_{2}=16, \sigma_{2}^{2}=0.000441$ and $\sigma_{2}=0.021$.

Further, using Eq. (7), we will have

$T=6 \sigma$

As a result, for the first component, we will have

$T_{1}=6 \times 0.022=0.132$.

For the second component, we will have:

$T_{2}=6 \times 0.021=0.126$.

Based on these tolerances, the tolerance and the technical characteristics of each component can be measured. Accordingly, the technical characteristics of the first component and those of the second one are $14 \pm 0.066$ and $16 \pm 0.063$, respectively. Now, the tolerance of the assembled set can be calculated as follows:

$$
\begin{aligned}
T & =\sqrt{T_{1}^{2}+T_{2}^{2}} \\
& =\sqrt{(0.132)^{2}+(0.126)^{2}}=0.182 .
\end{aligned}
$$

If we use additive relationship, and the tolerance of a component is equal, the tolerance of each component of the assembled set is expected to be $0.182 \mathrm{~mm}$, that is, more than the previous tolerance that was 0.129 . Consequently, it is obvious that more tolerance will be allocated to each component, using the probability relation and the central limit theorem. As a result, the allocation of more tolerance will facilitate the production of each component.

\section{Conclusion}

This study used the statistical method to design the tolerance of components. The results show that the use of this method will allow the allocation of more tolerance to each component in the process of assembling several components. Meanwhile, the assembled set can have the required performance. Designing tolerance by applying GLD for unknown distributions is the innovative contribution of this study. Due to its high flexibility, this type of distribution can be used both for estimation of parameters and design of tolerance. In addition to PM, there are many other methods used to estimate the GLD parameters. We can make sure that there is not a significant difference between the estimated values and the real values of parameters, using $\chi^{2}$.
Open Access This article is distributed under the terms of the Creative Commons Attribution 4.0 International License (http://crea tivecommons.org/licenses/by/4.0/), which permits unrestricted use, distribution, and reproduction in any medium, provided you give appropriate credit to the original author(s) and the source, provide a link to the Creative Commons license, and indicate if changes were made.

\section{References}

Armillotta A, Hartmann W (2015) Force analysis as a support to computer-aided tolerancing of planar linkages. Mech Mach Theory 93:11-25

Bigerelle M, Najjar D, Fournier B, Rupin N, Iost A (2006) Application of lambda distribution and bootstrap analysis to the prediction of fatigue lifetime and confidence intervals. Internet J Fatigue 28:223-236

Chandra MJ (2001) Statistical quality control. CRC Press LLC, 5-53

Delaney HD, Vargha A (2000) The effect on non-normality on student's two-sample t-test. The annual meeting of the American educational research Association, New Orlean

Dengiz B (1988) The generalized lambda distribution in simulation of m/m/1 queue systems. J Fac Eng Arch Gazi Univ 3:161-171

Devor RE, Tsong-How Chang, Sutherland JW (2007) Statistical quality design and control. Pearson Prentice hall, Upper saddle river, pp 366-404

Devroye L (1996) Random variant generation in one line of code. In: Charnes JM, Morrice DJ, Brunner DT, Swain JJ, eds. Proceedings of the Winter Simulation Conference. San Diego, CA, USA, December 8-11. Association for Computing Machinery, NY, pp 265-272

Filliben JJ (1975) The probability plot correlation coefficient test for normality. Technometrics 52:111-117

Fournier B, Rupin N, Bigerelle M, Najjar D, Iost A (2006) Application of the generalized lambda distribution in a statistical process control methodology. J Process Control 16:1087-1098

Fournier B, Rupin N, Bigerelle M, Najjar D, Iost A, Wilcox R (2007) Estimating the parameters of a generalized lambda distribution. Comput Stat Data Anal 51:2813-2835

Freimer M, Mudholkar S, Kollia G, Lin TC (1988) A study of the generalized Tukey Lambda family. Common Stat Theor Methods 17:3547-3567

Ganeshan R (2001) Are more supplier better? Generating the Gau and Ganeshan procedure. J Oper Res Soc 52:122-123

George J. Kaisarlis. (2012). A systematic approach for geometrical and dimensional tolerancing in reverse engineering, reverse engineering. Recent Advances and Applications

Gilchrist W (2000) Statistical Modeling with Quantile Function. CRC Press, Boca Raton

Ginsberg Robert H (2013) Outline of tolerancing from performance specification to toleranced drawings. Hughes Aircr Co Opt Eng 20(2): 175-180

Harrell FE, Davis CE (1982) A new distribution free quantile estimator. Biometrika 69:635-640

Hasenauer D (2013) Optical design tolerancing, a key to product cost reduction. Synopsys, Inc. 700 East Middlefield Road, Mountain View, CA 94043, www.synopsys.com

Hoaglin DC (1975) The small-sample variance of the Pitman location estimators. J Am Stat Assoc 52:880-888

Hoecke AV (2016) Tool risk setting in statistical tolerancing and its management in verification, in order to optimize customer's and supplier's risks. 14th CIRP Conference on Computer Aided Tolerancing (CAT), Procedia CIRP, 43, 250-255 
Jean-Marc J (2016) Process Tolerancing: a new approach to better integrate the truth of the processes in tolerance analysis and synthesis. 14th CIRP Conference on Computer Aided Tolerancing (CAT), Procedia CIRP, 43, 244-249

Joiner BL, Rosenblatt JR (1971) Some properties of the range in samples from Tukey's symmetric lambda distribution. J Am Stat Assoc 66:394

Karian ZA, Dudewicz EJ (1999) Fitting the generalized lambda distribution to data: a method based on percentiles. Commun Stat Simul Computat 28:793-819

Karian ZA, Dudewicz EJ (2000) Fitting statistical distributions. The generalized lambda distribution and generalized bootstrap methods. CRC Press, Boca Raton

Korn EL, Miothorne D, Graubard BJ (1997) Estimating interpolated percentiles from grouped data with large samples. J Off Stat 13:385-399

Lam H, Bowman KO, Shenton LR (1980) Remarks on the generalized Tukey's lambda family of distributions. In: Proc. ASA, Statist. Comput. Sec. Houston, Texas, August, 11-14, 134-139

Macko M, Ilić S, Jezdimirović M (2012) The influence of part dimensions and tolerance size to trigger characteristics. Strojniški vestnik J Mech Eng 58(6):411-415

Movahedi MM, Lotfi MR, Nayyeri M (2013) A solution to determining the reliability of products: using generalized lambda distribution. Res J Recent Sci 2(10):41-47

Najjar D, Bigerelle M, Lefebvre C, Lost A (2003) A new approach to predict the pit depth extreme value of a localized corrosion process. Isij 43:720-725

Nasser A, Aljazar L (2005) Generalized lambda distribution and estimation parameters. The Islamic University of Gaza, Deanery of Higher Studies, Faculty of Science, Department of Mathematics, Master of Science thesis, Supervised by Professor: Mohammed S. Elatrash

Nili Ahmadabadi M, Farjami Y, Bameni Moghadam M (2012) A process control method based on five-parameter generalized lambda distribution. Qual Quant, Springer Science + Business Media B.V. 46, 1097-1111

Ozturk A, Dale RF (1982) A study of fitting the generalized lambda distribution to solar radiation data. J Appl Meteorol 21:995-1004
Ramberg J, Schmeiser B (1974) An approximate method for generating asymmetric random variables. Commun ACM 17(2):78-82

Ramberg J, Dudewicz E, Tadikamalla P, E. Mykytka, E. (1979) A probability distribution and its uses in fitting data. Techno metrics 21(2):201-214

Rochan R, Upadhyay Ofodike A, Ezekoye (2008) Treatment of design fire uncertainty using quadrature method of moments. Five Saf J 43:127-139

Sampath Kumar R, Alagumurthi N, Ramesh R (2009) Optimization of design tolerance and asymmetric quality loss cost using pattern search algorithm. Int J Phys Sci 4(11):629-637

Sarabia JM (1996) A hierarchy of Lorenz curves based on generalized Tukeys lambda distribution. Econom Rev 16:305-320

Schmeiser BW, Deutsch SJ (1977) Quantile estimation from grouped data: the cell midpoint. Commun Stat Simul Computat 6:221-234

Shannon RR (2013) Tolerancing techniques. Optical Sciences Center University of Arizona Tucson, Arizona, pp 36.1-36.12

Shapiro SS, Wilk MB (1965) An analysis of variance test for normality (complete samples). Biometrika 52:591-611

Tarsitano A (2005) Estimation of the Generalized Lambda Distribution Parameters for Grouped Data. Taylor \& Francis Inc, Commun Stat Theory Methods 34:1689-1709

Tukey JW (1962) The future of data analysis. Ann Math Stat 33(1):1-67

Wadsworth HD, Stephens KS, Godfrrey AB (2002) Modern methods for quality control and improvement. Wiley, New York, pp 311-337

Weckenmann A, Hartmann W (2015) A model- and simulation-based approach for tolerancing and verifying the functional capability of micro/nano-structured workpieces. Measurement 76:70-79

Wheeler DL, Cavalier TM, Lehtihet EA (1999) An implicit enumeration approach to probabilistic tolerance allocation under conventional tolerance control. Int J Prod Res 37:3773

Zhang HC, Hook ME (1992) Tolerancing technique: the state-of-the art. Int J Prod Res 30:2111 